\title{
Variación local y geográfica de las infracomunidades de parásitos de la anchoveta Engraulis ringens en Chile
}

\author{
Local and geographical variation of parasite infracommunities of \\ the anchovy Engraulis ringens off Chile
}

\section{Mario George-Nascimento ${ }^{1}$ y David Moscoso $^{1}$}

\begin{abstract}
${ }^{1}$ Departamento de Ecología, Facultad de Ciencias, Universidad Católica de la Santísima Concepción, Casilla 297, Concepción, Chile.mgeorgen@ucsc.cl
\end{abstract}

\begin{abstract}
We compared the local and geographical variation of parasite infracommunities of the anchovy Engraulis ringens from 3 fishing areas off Chile (Caldera, Talcahuano and Valdivia), in 7 samples taken from different vessels. Body lengths of fish in the samples, richness, diversity and composition of parasite infracommunities revealed significant local variations among trawls, while only the composition of the parasite fauna showed significant geographical variations among fishing zones. Parasite infracommunities in hosts from Caldera were the most different and were characterized by ectoparasites, whereas those from Talcahuano and Valdivia harboured more endoparasites, mostly digeneans. These results confirm the existence of 2 stocks on the Chilean coast. This study is also the first report of parasitism in anchovies from Valdivia.
\end{abstract}

Key words: Ecological patterns, local variability, biological markers, ecological stocks

\section{INTRODUCCIÓN}

Aunque los estudios parasitológicos han sido ampliamente utilizados en la identificación de stocks y patrones migratorios de peces (Williams et al. 1992, MacKenzie 2002, MacKenzie et al. 2008), el progreso en el entendimiento de los procesos que originan patrones en las comunidades de parásitos requiere que se compare la magnitud de las variaciones locales y entre zonas geográficas (Poulin \& Valtonen 2002), lo que sólo recientemente ha sido considerado, junto a las variaciones de corto plazo (Ferrer-Castelló et al. 2007, Chávez et al. 2007). De hecho, ninguno de los estudios hechos en Chile acerca del uso de parásitos como indicadores de stock de sus hospedadores ha evaluado si en un momento del tiempo, la variación de las infracomunidades de parásitos entre cardúmenes de una misma localidad es o no de similar magnitud a la variación entre zonas geográficas. Una de las ventajas de utilizar los parásitos como marcadores biológicos es que pueden servir para identificar subpoblaciones o stocks ecológicos donde los estudios genéticos no pueden hacerlo (MacKenzie 2002, MacKenzie et al. 2008). Sin embargo, su correcto empleo requiere considerar aspectos que usualmente se desconocen, tales como la longevidad de los parásitos (Lester \& MacKenzie 2009).

La anchoveta Engraulis ringens es una especie de pez de hábitos pelágicos que habita en la zona nerítica del Océano Pacífico Sudoriental, y cuya distribución geográfica abarca desde Ecuador en el norte, hasta la isla de Chiloé en el sur de Chile. Su promedio de vida alcanzaría entre 4 y 5 años, y forma cardúmenes que se distribuyen entre los $50 \mathrm{~m}$ de profundidad durante el día y en la superficie durante la noche, a una distancia no superior a las 20 millas náuticas desde la costa (Serra et al. 1979). Además, es presa de peces, mamíferos marinos y aves marinas (Muck \& Pauly 1987, Cubillos et al. 2007, Hückstädt et al. 2007).

Entre los estudios en comunidades de parásitos de engráulidos en Sudamérica se cuenta el de Engraulis anchoita (Hubbs \& Marini, 1935) en Argentina (Timi 2003, Timi \& Poulin 2003) que mostró que los ensambles de parásitos presentaban un marcado gradiente latitudinal. Otro estudio mostró que las infracomunidades de parásitos metazoos de Anchoa tricolor (Agassiz, 1829) en una localidad de la costa de Brasil estaban dominadas por endoparásitos larvales existiendo correlaciones entre la abundancia, dominancia y riqueza con el tamaño corporal del hospedador (Tavares et al. 2005).

En este estudio se compara la variación local de las infracomunidades de parásitos de la anchoveta Engraulis ringens (Jenyns, 1842), con la variación geográfica de ellas, en 3 zonas de pesca de la costa de Chile que están ampliamente separadas (Caldera, Talcahuano y Valdivia). Con esto se amplía el registro geográfico del parasitismo en esta especie en la costa de Chile, ya que por primera 
vez se examinan ejemplares de la costa de Valdivia. Los antecedentes del parasitismo de esta especie en Chile señalan que habrían 2 stocks, uno entre Arica y Coquimbo, y otro entre Valparaíso y Talcahuano (Valdivia et al. 2007), y que la variabilidad local de las comunidades componentes de parásitos sería baja en el norte de Chile (Chávez et al. 2007). Sin embargo, el único estudio genético realizado en esta especie concluye que las anchovetas capturadas en Iquique y en Talcahuano son parte de una población panmíctica (Ferrada et al. 2002).

\section{Materiales y MÉtodos}

Se examinaron parasitológicamente 221 anchovetas provenientes de 3 zonas de pesca en Chile: Caldera ( $\left.27^{\circ} 00^{\prime} \mathrm{S}, 70^{\circ} 45^{\prime} \mathrm{W}\right)$, Talcahuano ( $36^{\circ} 43^{\prime} \mathrm{S}, 7^{\circ} 07^{\prime} \mathrm{W}$ ) y Valdivia (39³8'S, $73^{\circ} 05^{\prime} \mathrm{W}$ ), obtenidas de 7 muestras, tomadas desde la bodega de distintas embarcaciones artesanales, en fechas vecinas entre fines de junio y mediados de julio del 2005. En Caldera, el día 28/06/2005 se obtuvieron 2 muestras compuestas de 31 y 28 ejemplares cada una. En Talcahuano se tomaron 3 muestras, cada una compuesta por 30 ejemplares: una fue tomada el 14/06/2005 y las otras 2 el 15/06/2005. En Valdivia se tomaron 2 muestras, una el 07/07/2005 de 33 anchovetas, y la otra el 15/07/2005 con 39 ejemplares. Se infiere que estas muestras provienen de distintos cardúmenes de una misma zona de pesca en un momento del tiempo, y que representan la variabilidad local.

Los ejemplares fueron congelados a $-18^{\circ} \mathrm{C}$ en bolsas individuales debidamente rotuladas con su localidad de procedencia y fecha de captura. En el laboratorio, y una vez descongelados, se determinó la longitud total (mm) de cada hospedador. Cada individuo fue examinado externamente bajo lupa para localizar ectoparásitos en la piel, cavidad bucal y cámara branquial. La inspección para endoparásitos fue realizada mediante la disección de las vísceras, las cuales fueron llevadas a chorro de agua a presión para la retención y la recolección de los parásitos en una malla de 0,5 mm. Luego, los parásitos encontrados fueron determinados taxonómicamente hasta el nivel más bajo posible, y fijados en formalina al $5 \%$.

La magnitud de cada parasitosis fue descrita mediante la prevalencia y la abundancia (Bush et al. 1997). Entre los descriptores infracomunitarios considerados, la abundancia total de parásitos fue medida como el número de individuos parásitos por hospedador parasitado, independiente de la identidad específica del parásito. La riqueza fue medida como el número de taxa por hospedador parasitado, y la diversidad infracomunitaria (loge) calculada con el índice de Brillouin (Magurran 1988), y determinada sólo en los hospedadores que albergaban algún parásito.

La composición de las infracomunidades fue resumida mediante una ordenación realizada con un análisis de correspondencia efectuado sobre la raíz cuadrada de la abundancia relativa de los taxa en las infracomunidades (transformación de Hellinger, ver Legendre \& Gallagher 2001), y usando el método de promedios recíprocos (Jongman et al. 1995). La composición de las infracomunidades fue expresada como el puntaje de cada una en el primer eje de la ordenación. En dichas ordenaciones se incluyeron 140 anchovetas (de 152 parasitadas) y 8 taxa parasitarios, los que daban cuenta del 96,8\% del total de parásitos recolectados, ya que sólo se consideraron los hospedadores que albergaban parásitos, y los taxa que estaban en al menos 5 anchovetas en el total de las muestras. Este criterio numérico permitió eliminar los taxa de mínima ocurrencia, ya que afectan en demasía la dimensionalidad mínima y los puntajes de las ordenaciones, en especial en infracomunidades muy ‘vacías' (Jongman et al. 1995).

El efecto de la longitud total de los hospedadores sobre la abundancia total, la riqueza, la diversidad y la composición de las infracomunidades fue evaluado con análisis de regresión lineal. Luego, y toda vez que dicha variable mostró efectos significativos, su efecto fue corregido mediante el uso de los residuos. La evaluación de la significancia estadística de las variaciones locales consistió en análisis anidados de la varianza de la longitud total, abundancia total, riqueza, diversidad y composición de las infracomunidades. Se usaron pruebas de Chicuadrado para evaluar la significancia estadística de las diferencias en prevalencia total (Gotelli \& Ellison 2004).

\section{RESUlTAdOS Y DISCUSIÓN}

Del total de anchovetas ( $\mathrm{n}=221$ ), el 68,7\%, tenía parásitos; 33,9\% de los 59 ejemplares recolectados en Caldera, 75,6\% de los 90 ejemplares recolectados en Talcahuano, y el 88,9\% de los 72 ejemplares de Valdivia. Sólo en Talcahuano se encontraron diferencias significativas entre muestras al comparar la prevalencia total del parasitismo $\left(\chi^{2}=12,39\right.$, g. l. $=2 ; P=0,002$, Tabla 1). La prevalencia total no mostró diferencias significativas entre muestras en Caldera y en Valdivia $\left(0,252<\chi^{2}<0,675\right.$, g. l. $=1 ; 0,411<P<0,616$, Tabla 1). Entre zonas de pesca, la prevalencia total fue significativamente mayor en Valdivia que en Talcahuano 


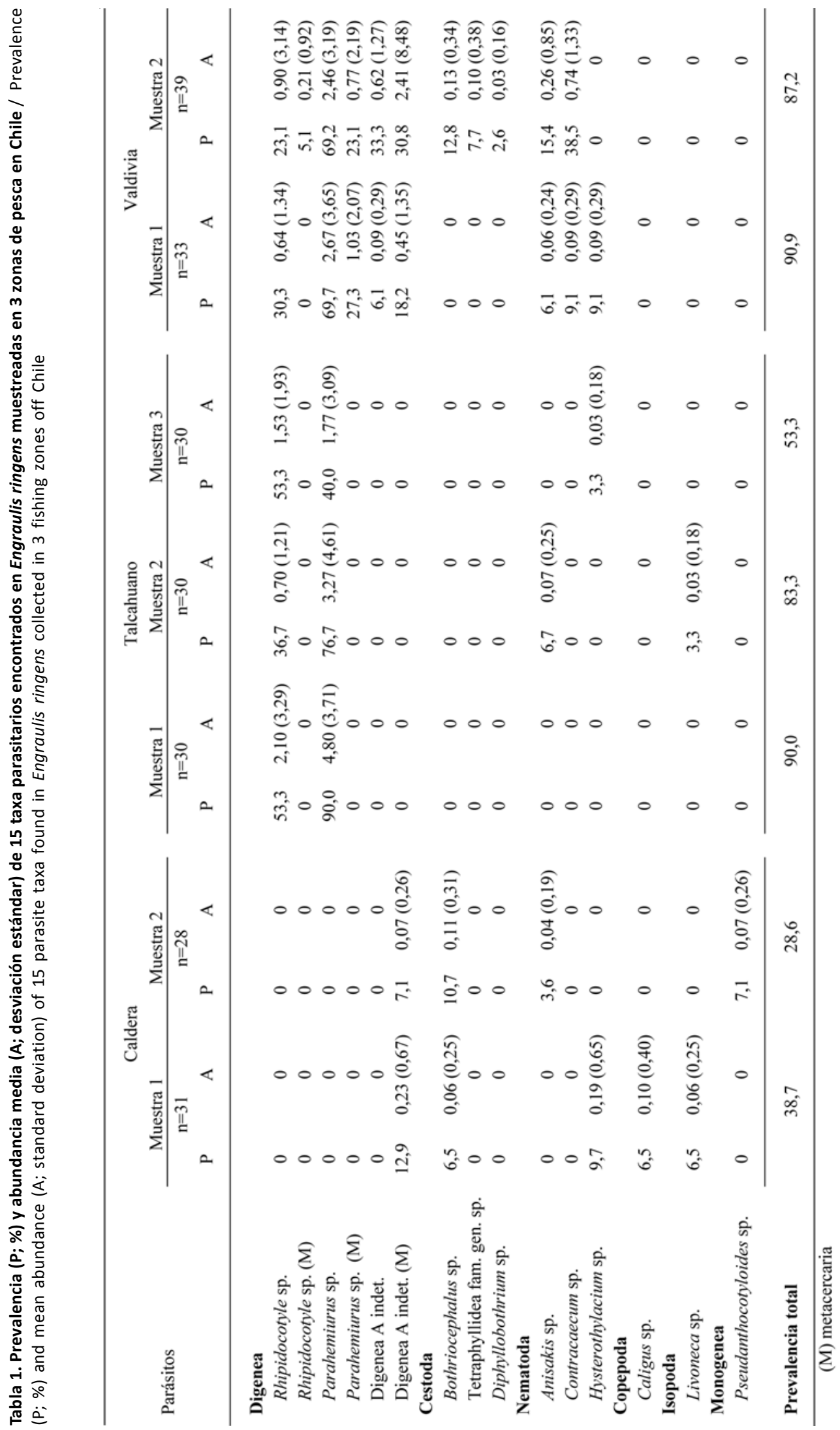


$\chi^{2}=4,71$, g. l. $\left.=1 ; P<0,04\right)$ y Caldera $\left(\chi^{2}=42,62\right.$, g. $1 .=1$; $P<0,001)$, y mayor en Talcahuano que en Caldera $\left(\chi^{2}=\right.$ $25,58$, g. $1 .=1, P<0,001$, Tabla 1$)$. Se recolectó un total de 962 individuos parásitos pertenecientes a 15 taxa nominales de los cuales 4 correspondieron a endoparásitos adultos, 8 a endoparásitos larvales y 3 a ectoparásitos (Tabla 1).

La longitud total de las anchovetas fluctuó entre 105 y $183 \mathrm{~mm}$, y sus variaciones resultaron ser significativas tanto entre muestras dentro de las zonas de pesca $\left(\mathrm{F}_{(4,214)}\right.$ =7,97; $P<0,001)$, como entre las zonas de pesca $\left(\mathrm{F}_{(2,4)}=\right.$ $14,11 ; P=0,010)$. Los ejemplares de mayor tamaño correspondieron a las muestras tomadas en Valdivia (Tabla 2).

La longitud total de los hospedadores reveló ser una variable de efectos significativos sólo sobre las variaciones de la diversidad $\left(\mathrm{r}^{2}=0,037, \mathrm{~F}_{(1,150)}=5,80, P=\right.$ $0,017)$, y sobre la composición de las infracomunidades $\left(\mathrm{r}^{2}=0,076, \mathrm{~F}_{(1,139)}=11,45, P=0,001\right)$. Luego de que estos 2 últimos descriptores comunitarios fueran corregidos por la longitud total de los hospedadores, los análisis de la varianza anidados mostraron variaciones locales significativas en la riqueza $\left(\mathrm{F}_{(4,145)}=5,89 ; P=0,002\right)$, en la diversidad $\left(\mathrm{F}_{(4,145)}=4,99 ; P=0,001\right)$ y en la composición de las infracomunidades $\left(\mathrm{F}_{(4,133)}=4,45 ; P<0,002\right)$ entre muestras. La composición de las infracomunidades fue el único descriptor que mostró variaciones significativas entre zonas de pesca (composición: $\mathrm{F}_{(2,4)}=8,10 ; P=0,039$, riqueza: $\mathrm{F}_{(2,4)}=1,61 ; P=0,307$, diversidad: $\mathrm{F}_{(2,4)}=2,89 ; P$ $=0,167$, Tabla 2). Las muestras provenientes de Caldera eran las más diferentes.
La representación gráfica del primer gradiente composicional de las infracomunidades (valor propio = 0,756 ), en relación con la longitud total de las anchovetas mostró que las infracomunidades de los hospedadores de las muestras de Talcahuano y Valdivia se agruparon entre las coordenadas correspondientes a Parahemiurus. sp. y Riphidocotyle sp., en tanto que la mayoría de las infracomunidades de los hospedadores de Caldera se dispersaron entre las coordenadas correspondientes a Digenea A indet. (M) e Hysterothylacium sp. (Fig. 1).

Estos resultados señalan que la variación local de las infracomunidades de parásitos de la anchoveta puede ser de magnitud no despreciable y por consiguiente, no debiera ser ignorada al momento de efectuar futuros estudios. Por ello, es posible que en zonas en que las infracomunidades son más ricas, como la zona sur de Chile, no sea suficiente sólo una muestra para representar adecuadamente la variabilidad en la composición a nivel de comunidad componente, como fue propuesto por Chávez et al. (2007) para la zona norte de Chile (entre Iquique y Coquimbo). Se sostiene que el diseño de muestreo aquí empleado permite abordar de forma más robusta las preguntas concernientes a la existencia de stocks ecológicos ya que se puede evaluar la importancia de las variaciones locales del parasitismo, y no sólo entre zonas geográficas. Cabe resaltar que Engraulis ringens es de corta vida, lo que dificulta el empleo de los parásitos como marcadores. De hecho, muchos peces no presentan parásitos, o bien son escasos, y los parásitos de infección acumulativa son también escasos. A pesar de ello, las infracomunidades de parásitos de la anchoveta tienen características cuantitativas y cualitativas distintivas según la zona de pesca. La inclusión de muestras tomadas

Tabla 2. Promedio \pm desviación estándar de la longitud total $(\mathrm{mm})$, abundancia total, riqueza, diversidad y composición de las infracomunidades de párásitos en E. ringens de 3 zonas de pesca en Chile / Mean \pm standard deviation of total body length $(\mathrm{mm})$, total abundance, richness, diversity and composition of parasite infracommunities in E. ringens in 3 fishing zones off Chile

\begin{tabular}{lcccccr}
\hline Zonas & Muestras & Longitud & Abundancia & Riqueza & Diversidad & Composición \\
\hline Caldera & 1 & $145 \pm 12$ & $0,64 \pm 1,11$ & $0,41 \pm 0,56$ & $0,03 \pm 0,13$ & $235 \pm 80,6$ \\
& 2 & $143 \pm 16$ & $0,28 \pm 0,46$ & $0,28 \pm 0,46$ & $0,01 \pm 0,01$ & $190 \pm 30,9$ \\
\multirow{2}{*}{ Talcahuano } & 1 & $130 \pm 8$ & $3,33 \pm 4,14$ & $0,96 \pm 0,96$ & $0,34 \pm 0,18$ & $52 \pm 41,8$ \\
& 2 & $131 \pm 13$ & $4,06 \pm 4,95$ & $1,23 \pm 0,77$ & $0,18 \pm 0,22$ & $29 \pm 49,3$ \\
& 3 & $123 \pm 14$ & $6,90 \pm 6,36$ & $1,43 \pm 0,67$ & $0,26 \pm 0,24$ & $17 \pm 17,6$ \\
Valdivia & 1 & $158 \pm 8$ & $5,12 \pm 5,35$ & $1,33 \pm 0,69$ & $0,31 \pm 0,28$ & $103 \pm 89,4$ \\
& 2 & $157 \pm 7$ & $8,61 \pm 17,97$ & $1,92 \pm 1,24$ & $0,55 \pm 0,38$ & $96 \pm 76,5$ \\
\hline
\end{tabular}




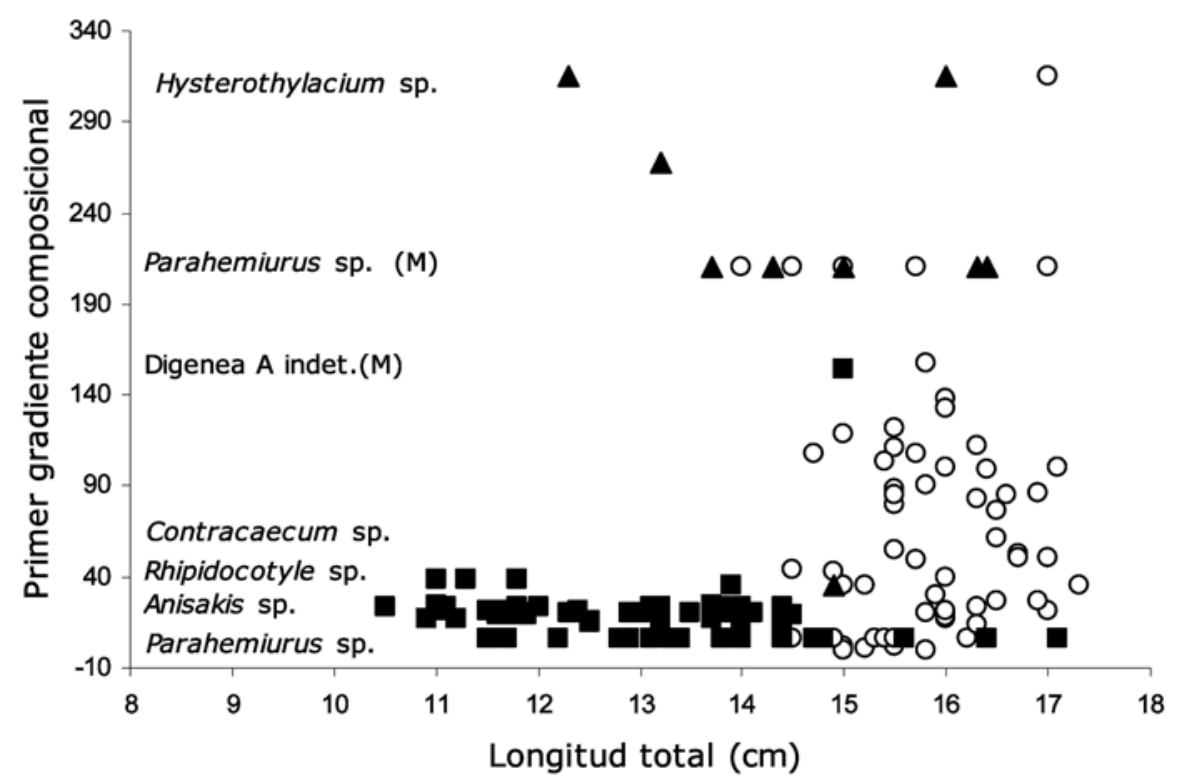

Figura 1. Relación entre el primer gradiente composicional de las infracomunidades de parásitos en el primer eje del análisis de correspondencia, con la longitud total de las anchovetas $(\mathrm{cm})$ de acuerdo a las zonas de pesca; Caldera $(\boldsymbol{\Delta})$, Talcahuano $(\square)$ y Valdivia (O). Los puntajes y los nombres de los taxa parasitarios son indicados a lo largo del primer gradiente composicional / Relationship between the first compositional gradient of parasites infracommunities obtained from a correspondence analysis with total body length of the anchovies $(\mathrm{cm})$ according to fishing zones; Caldera ( $\mathbf{\Delta})$, Talcahuano $(\mathbf{\square})$ and Valdivia (O). The scores and names of the parasite taxa are indicated along the first compositional axis

en las vecindades de Valdivia permitió revelar que las anchovetas de dicha zona albergan infracomunidades más ricas en especies que las de Caldera, aunque no son muy diferentes a las de Talcahuano. Futuros estudios con mayor resolución taxonómica de los digeneos podrían apoyar la existencia de un stock más austral que el propuesto por Valdivia et al. (2007). En suma, el hallazgo de diferencias geográficas en la composición de las infracomunidades permite reforzar el hecho de que habrían al menos 2 stocks de E. ringens, según lo propuesto por Valdivia et al. (2007). Cabe mencionar que la presencia de Pseudanthocotyloides sp. y Caligus sp. en anchovetas de la zona norte de Chile permitieron a Valdivia et al. (2007) discriminar esta zona de la zona central (entre Valparaíso y Talcahuano), lo que se confirma con los resultados aquí obtenidos.

En términos generales, los resultados obtenidos concuerdan con lo señalado por Timi \& Poulin (2003) en Engraulis anchoita de Argentina, apoyando un gradiente latitudinal de condiciones ambientales y su influencia tanto en la distribución del zooplancton como de hospedadores definitivos que determinarían las diferencias de los ensambles de parásitos entre las poblaciones de hospedadores, siendo estas diferencias reforzadas por el comportamiento migratorio de los hospedadores (Timi 2003). Futuros estudios en E. ringens, con mayor resolución taxonómica de los digeneos y realizados en una secuencia temporal, podrían reforzar el hallazgo de que las infecciones por metacercarias son indicadoras de anchovetas 'sureñas', en forma similar a lo registrado por Campbell et al. (2007) en Clupea harengus.

\section{Agradecimientos}

Los autores agradecen el financiamiento del proyecto FONDECYT 1050528, y las sugerencias de los revisores.

\section{LITERATURA CITADA}

Bush A, K Lafferty, J Lotz \& A Shostak. 1997. Parasitology meets ecology on its own terms: Margolis et al. revisited. The Journal of Parasitology 83: 575-583.

Campbell N, MA Cross, JC Chubb, CO Cunningham, EMC Hatfield \& K MacKenzie. 2007. Spatial and temporal variations in parasite prevalence and infracommunity structure in herring (Clupea harengus L.) caught to the west of the British Isles and in the North and Baltic seas: implications for fishery science. Journal of Helminthology 81: 137-146. 
Chávez R, I Valdivia \& M Oliva. 2007. Local variability in metazoan parasites of the pelagic fish species, Engraulis ringens: implications for fish stock assessment using parasites as biological tags. Journal of Helmintology 81: 113-116

Cubillos L, C Alarcón \& H Arancibia. 2007. Selectividad por tamaño de las presas en merluza común (Merluccius gayi gayi), zona centro-sur de Chile (1992-1997). Investigaciones Marinas 35: 55-69.

Ferrada S, K Hernández, R Montoya \& R Galleguillos. 2002. Estudio poblacional del recurso anchoveta (Engraulis ringens Jenyns 1842) (Clupeiformes, Engraulidae), mediante análisis de ADN. Gayana 66: 243-248.

Ferrer-Castelló E, J Raga \& F Asnar. 2007. Parasites as fish population tags and pseudoreplication problems: the case of striped red mullet Mullus surmuletus in the Spanish Mediterranean. Journal of Helminthology 81: 169-178.

Gotelli NJ \& AM Ellison. 2004. A primer of ecological statistics, 510 pp. Sinauer Associates, Sunderland.

Hückstädt LA, CP Rojas \& T Antezana. 2007. Stable isotope analysis reveals pelagic foraging by the Southern sea lion in central Chile. Journal of Experimental Marine Biology and Ecology 347: 123-133.

Jongman RHG, CJF Ter Braak \& OFR Van Tongeren. 1995. Data analysis in community and landscape ecology, 299 pp. Cambridge University Press, New York.

Legendre P \& ED Gallagher. 2001. Ecologically meaningful transformations for ordination of species data. Oecologia 129: 271-280.

Lester R \& K MacKenzie. 2009. The use and abuse of parasites as stock markers for fish. Fisheries Research 97: $1-2$.

MacKenzie K. 2002. Parasites as biological tags in population studies of marine organisms: an update. Parasitology 124: S153-S163.

MacKenzie K, N Campbell, S Mattiucci, P Ramos, AL Pinto \& P Abaúnza. 2008. Parasites as biological tags for stock identification of Atlantic horse mackerel Trachurus trachurus. Fisheries Research 89: 136-145.
Magurran AE. 1988. Ecological diversity and its measurement, 178 pp. Princeton University Press, New Jersey.

Muck P \& D Pauly. 1987. Monthly anchoveta consumption of guano birds 1953 to 1983 . In: Pauly D \& I Tsukayama (eds). The Peruvian anchoveta and its upwelling ecosystem: three decades of change. ICLARM Studies and Reviews 15: 219-233.

Poulin R \& E Valtonen. 2002. The predictability of helminth community structure in space: a comparison of fish populations from adjacent lakes. International Journal for Parasitology 32: 1235-1243.

Serra R, M Aguayo, O Rojas, J Cañón \& F Inostroza. 1979. Anchoveta Engraulis ringens (Jenyns) Teleostomi Clupeiformes Engraulidae. En: Estado actual de las principales pesquerías nacionales. Bases para un desarrollo pesquero. CORFO-IFOP 18: 1-52. CORFO, Santiago de Chile.

Tavares L, J Luque \& A Bicudo. 2005. Community ecology of metazoan parasites of the anchovy Anchoa tricolor (Osteichthyes: Engraulidae) from the coastal zone of the state of Rio de Janeiro, Brazil. Brazilian Journal of Biology 65: 533-540.

Timi J. 2003. Parasites of Argentine anchovy in the southwest Atlantic: latitudinal patterns and their use for discrimination of host populations. Journal of Fish Biology 63: 90-107.

Timi J \& R Poulin. 2003. Parasite community structure within and across host populations of a marine pelagic fish: how repeatable is it? International Journal for Parasitology 33: 1353-1362.

Valdivia I, R Chávez \& M Oliva. 2007. Metazoan parasites of Engraulis ringens as tools for stock discrimination along the Chilean coast. Journal of Fish Biology 70: 1-8.

Williams H, K Mackenzie \& A McCarthy. 1992. Parasites as biological indicators of the population biology, migrations, diet, and phylogenetics of fish. Reviews in Fish Biology and Fisheries 2: 144-176.

Recibido el 25 de octubre de 2012 y aceptado el 17 de enero de 2013

Editor Asociado: Gabriela Muñoz C. 